Pacific Journal of Mathematics

THE INVERSE OF A CONTINUOUS ADDITIVE FUNCTIONAL 


\title{
THE INVERSE OF A CONTINUOUS ADDITIVE FUNCTIONAL
}

\author{
JEAN-MARIE RoLIN
}

Let $X$ be a standard process and $A$ be a continuous additive functional of $X$. The inverse of $A$ is defined by $\tau_{t}=$ $\inf \left\{s \cdot A_{s}>t\right\}$. The aim of this paper is to prove that the process $\tau$ has conditionally independent increments with respect to the $\sigma$-algebra generated by the time changed process $\hat{X}_{t}=$ $X_{\tau_{i}}$. However these increments are not necessarily stationary. Another interesting result is derived: the continous part of the process $\tau$ is a continuous additive functional of the process $\hat{X}$.

The existence of regular conditional probabilities permits to consider the process $\tau$ as an additive process and under a necessary and sufficient condition, it is in fact a Levy process with increasing paths. The general theory of such processes is then used to obtain a Levy representation of the jumps of the process $\tau$.

1. Introduction. Let us consider a standard process $X=$ $\left(\Omega, \mathcal{M} ; E, \mathscr{E}, \Delta ; \mathcal{M}_{t}, X_{t}, \theta_{t}, P^{x}\right)$ and a continuous additive functional (C.A.F.) of $X$. We refer to [1] for all the notations and definitions of such concepts.

It is well known in the theory of the Lebesgue-Stieltjes integral that if we define

$$
\tau_{t}=\inf \left\{s: A_{s}>t\right\}
$$

then for all nonnegative Borel functions $f$ on $[0, \infty]$ vanishing at infinity, the following formula holds

$$
\int_{0}^{\infty} f(t) d A_{t}=\int_{0}^{\infty} f\left(\tau_{t}\right) d t
$$

The aim of this paper is to investigate some of the probabilistic properties of this "inverse" of the continuous additive functional $A$.

It is easy to see that for each $s, \tau_{s}$ is a stopping time for $X$ and it is known that under some additional assumptions, the time changed process $X_{\tau_{t}}$ is a standard process (see [1]-V-2, 11, and [3]).

Some important results have been established by Blumenthal and Getoor in the case where the fine support of $A$ consists of a single point 
$x_{0}$. That is the theory of local times that shows in particular that the process $\left(\tau_{t}, P^{x_{0}}\right)$ is more or less equivalent to a subordinator. For a precise statement of this theorem, refer to [1]-V-3.

We are now going to show that in the general case, the process $\tau$ has conditionally independent increments with respect to the $\sigma$-algebra generated by the process $\hat{X}$.

II. The conditioning. Let $X=\left(\Omega, \mathcal{M}, E, \mathscr{E}, \Delta ; X_{t}, \mathcal{M}_{t}, \theta_{t}, P^{x}\right)$ be a standard process with lifetime $\xi$ and $A$ be a continuous additive functional of $X$. We will suppose that for all $\omega$ in $\Omega$, the functions $t \rightarrow A_{t}(\omega)$ are continuous on $[0, \infty]$ and the paths functions $t \rightarrow X_{t}(\omega)$ are right continuous on $[0, \infty]$ and have left-hand limits on $[0, \xi(\omega))$. Let us introduce some notations. We will write

$$
\begin{gathered}
\hat{X}_{t}=X_{\tau_{t}} \\
\hat{\theta}_{t}=\theta_{\tau_{t}} \\
\tilde{\mathscr{F}}_{t}=\mathscr{F}_{\tau_{t}}
\end{gathered}
$$

$\hat{\mathscr{F}}_{t}^{0}$ and $\hat{\mathscr{F}}^{0}$ will have their usual meanings relative to the process $\hat{X}$ and $\hat{\mathscr{F}}_{t}$ and $\hat{\mathscr{F}}$ will be their respective completions by the family $P^{\mu}$ as sub- $\sigma$-algebras of $\mathscr{F}$. To make this precise, $A$ will be in $\hat{\mathscr{F}}_{t}(\hat{\mathscr{F}})$ if for each finite measure $\mu$ on $\left(E_{\Delta}, \mathscr{E}_{\Delta}\right)$ there exist sets $B_{\mu}$ in $\hat{\mathscr{F}}_{t}^{0}\left(\hat{\mathscr{F}}^{0}\right)$ and $N_{\mu}$ in $\mathscr{F}^{0}$ such that $P^{\mu}\left(N_{\mu}\right)=0$ and $B_{\mu}-N_{p} \subset A \subset B_{\mu} \cup N_{\mu}$. Let us remark that $Y$ in $\mathscr{F}$ will be in $\hat{\mathscr{F}}_{t}(\hat{\mathscr{F}})$ if for each finite measure $\mu$ on $\left(E_{\Delta}, \mathscr{E}_{\Delta}\right)$ there exists $Z_{\mu}$ in $\hat{\mathscr{F}}_{t}(\hat{\mathscr{F}})$ such that $Y=Z_{\mu}$ almost surely $P^{\mu}$.

It follows immediately from these definitions that $\hat{\mathscr{F}}_{t}$ is contained in $\tilde{\mathscr{F}}_{t}$ and $\hat{\mathscr{F}}$ is contained in $\mathscr{F}_{\text {. }}$ Since by definition, $\hat{X}_{t}$ is in $\hat{\mathscr{F}}^{0} \mid \mathscr{E}_{\Delta}$, it is clear that $\hat{X}_{t}$ is in $\hat{\mathscr{F}} \mid \mathscr{E}_{\Delta}^{*}$ for each $t$, where $\mathscr{E}_{\Delta}^{*}$ denotes the $\sigma$-algebra of universally measurable sets over $\left(E_{\Delta}, \mathscr{E}_{\Delta}\right)$. It is also easy to see that $\hat{\theta}_{t}$

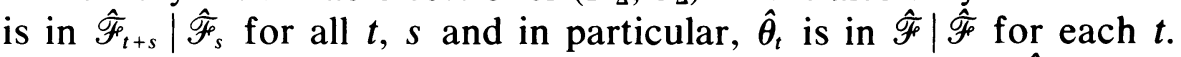

Now if we consider the lifetime $\xi$ of the process $\hat{X}$, i.e. $\xi=$ $\inf \left\{t: \hat{X}_{t}=\Delta\right\}$ we note that

$$
\xi=A_{\xi}=A_{\infty} \quad \text { a.s. }
$$

since $\left\{\hat{X}_{t}=\Delta\right\}=\left\{\tau_{t} \geqq \xi\right\}=\left\{A_{\xi} \leqq t\right\}$.

We are now ready to state some lemmas. The simplicity of their proofs will permit us to omit them.

Lemma 2.1. Let $T$ be a $\left\{\tilde{\mathscr{F}}_{t}\right\}$ stopping time. Then $\tau_{T}$ is a $\left(\mathscr{F}_{t}\right\}$ stopping time and $\tilde{\mathscr{F}}_{T}=\mathscr{F}_{T_{T}}$. Moreover for all $t$,

$$
\tau_{T+t}=\tau_{T}+\tau_{t} \circ \hat{\theta}_{T} \quad \text { a.s. }
$$


and

$$
A_{\tau T}=T \text { on }\{T<\hat{\xi}\}
$$

Lemma 2.2. Let $T$ be a $\left\{\mathscr{F}_{t}\right\}$ stopping time. Then $A_{T}$ is a $\left\{\tilde{\mathscr{F}}_{t}\right\}$ stopping time and $\mathscr{F}_{T}$ is contained in $\tilde{\mathscr{F}}_{A_{T}}$. Moreover for all $t$,

$$
\tau_{A_{\Gamma+t}}=T+\tau_{t} \circ \theta_{T} \quad \text { a.s. on } \quad\left\{A_{T}<\infty\right\} \text {. }
$$

LeMmA 2.3. Let $Y$ be in $\hat{\mathscr{F}}$ and $T$ be a $\left\{\mathscr{F}_{t}\right\}$ stopping time. Then

$$
Y \circ \hat{\theta}_{A_{T}}=Y \circ \theta_{T} \quad \text { a.s. on }\left\{A_{T}<\infty\right\} .
$$

In particular, if we take $T \equiv 0$, then

$$
Y=Y \circ \hat{\theta}_{0} \quad \text { a.s. }
$$

Let us turn now to some considerations related to the support of the continuous additive functional $A$. We will denote it by $F$. By definition

$$
F=\left\{x \in E: P^{x}\left(\tau_{0}=0\right)=1\right\}
$$

It is known (see [1]-V-3) that $F$ is a nearly Borel set which is finely perfect, i.e. the set of regular points for $F$ is precisely $F$, and that is a consequence of the fact that

$$
T_{F}=\tau_{0} \quad \text { a.s. }
$$

where $T_{F}$ is the hitting time of the set $F$. Moreover for all $x$ in $E_{\Delta}$,

$$
P^{x}\left[\hat{X}_{t} \notin F \quad \text { for some } \quad t<\hat{\xi}\right]=0 \text {. }
$$

Using this result, we can and we will from now on, suppose that the process $\hat{X}$ lives on $F \cup\{\Delta\}$. It is also easy to prove that for all $\left\{\mathscr{F}_{t}\right\}$ stopping times $T$,

$$
\left\{X_{T} \in F\right\}=\left\{\tau_{0} \circ \theta_{T}=0\right\} \text { a.s. }
$$

In the sequel, we will have to deal with expressions of the form

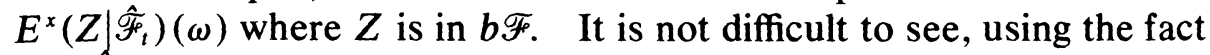
that $\hat{\mathscr{F}}_{t}^{0}$ is countably generated and the martingale convergence theorem, that we can choose a version which is jointly measurable in $x$ and 
$\omega$. More precisely: if $Z$ is in $b \mathscr{F}$ and $t \leqq \infty$, then there exists $Z_{t}^{x}(\omega)$ in $b \mathscr{E}_{\Delta}^{*} \otimes \hat{\mathscr{F}}_{t}$ such that for all $x$ in $E_{\Delta}, E^{x}\left(Z \mid \hat{\mathscr{F}}_{t}\right)=Z_{t}^{x}$ a.s. $P^{x}$. Since $E^{x}\left(Z \mid \hat{\mathscr{F}}_{t}\right)$ is only defined a.s. $P^{x}$, we will always suppose when writing expressions such as $E^{x}\left(Z \mid \hat{\mathscr{F}}_{t}\right)(\omega)$ that it is jointly measurable in $x$ and $\omega$.

We now come to an important lemma.

Lemma 2.4. Let $Z_{1}^{x}(\omega), Z_{2}^{x}(\omega)$ be in $b \mathscr{E}_{\Delta}^{*} \otimes \hat{\mathscr{F}}$ and such that, for each $x$ in $F \cup\{\Delta\}, Z_{1}^{x}=Z_{2}^{x}$ a.s. $P^{x}$. Then

$$
Z_{1}^{\hat{X}_{0}}=Z_{2}^{\hat{X}_{0}} \text { a.s. }
$$

Proof. Clearly $Z_{i}^{\hat{\chi}_{0}}$ is in $b \hat{\mathscr{F}}$ and by the preceding lemma, for all finite measures $\mu$ on $\left(E_{\Delta}, \mathscr{E}_{\Delta}\right)$ and for all $A$ in $\hat{\mathscr{F}}$

$$
E^{\mu}\left(1_{A} Z_{i}^{\hat{X}_{0}}\right)=E^{\mu} E^{\hat{X}_{0}}\left(1_{A} Z_{i}^{\hat{X}_{0}}\right)=\int_{F \cup\{\Delta\}} E^{x}\left(1_{A} Z_{i}^{\hat{X}_{0}}\right) P^{\mu}\left(\hat{X}_{0} \in d x\right) .
$$

Now if $x$ is in $F, \tau_{0}=0$ a.s. $P^{x}$ and $\hat{X}_{0}=x$ a.s. $P^{x}$. If $x=\Delta, \tau_{0}=\infty$ a.s. $P^{\Delta}$ and $\hat{X}_{0}=\Delta$ a.s. $P^{\Delta}$. Hence for all $x$ in $F \cup\{\Delta\}$,

$$
E^{x}\left(1_{A} Z_{1}^{\hat{X}_{0}}\right)=E^{x}\left(1_{A} Z_{1}^{x}\right)=E^{x}\left(1_{A} Z_{2}^{x}\right)=E^{x}\left(1_{A} Z_{1}^{\hat{X}_{0}}\right)
$$

and

$$
E^{\mu}\left(1_{A} Z_{1}^{\hat{X}_{0}}\right)=E^{\mu}\left(1_{A} Z_{2}^{\hat{X}_{0}}\right) .
$$

That implies that

$$
Z_{1}^{\hat{X}_{0}}=Z_{2}^{\hat{X}_{0}} \quad \text { a.s. } \quad P^{\mu},
$$

and the conclusion holds since $\mu$ is arbitrary.

In the sequel, we will usually omit the $\omega$ 's when writing expressions such as $E^{\hat{X}_{t}\left(\hat{\theta}_{s} \omega\right)}\left[Z \mid \hat{\mathscr{F}}_{u}\right]\left(\hat{\theta}_{v} \omega\right)$. We will write

$$
\begin{aligned}
& E^{\hat{X}_{t}}\left[Z \mid \hat{\mathscr{F}}_{v}\right] \circ \hat{\theta}_{s}(\omega)=E^{\hat{X}_{t}\left(\hat{\theta}_{s} \omega\right)}\left[Z \mid \hat{\mathscr{F}}_{v}\right]\left(\hat{\theta}_{s} \omega\right) \\
& E^{\hat{X}_{t}}\left[Z \mid \hat{\mathscr{F}}_{v}\right]\left(\hat{\theta}_{s}\right)(\omega)=E^{\hat{X}_{t}(\omega)}\left[Z \mid \hat{\mathscr{F}}_{v}\right]\left(\hat{\theta}_{s} \omega\right) .
\end{aligned}
$$

For instance, we have almost surely

$$
E^{\hat{X}_{t}}\left(Z \mid \hat{\mathscr{F}}_{v}\right) \circ \hat{\boldsymbol{\theta}}_{s}=E^{\hat{X}_{t+s}}\left[Z \mid \hat{\mathscr{F}}_{v}\right]\left(\hat{\boldsymbol{\theta}}_{s}\right)
$$

We are now ready to state the main theorem of this section. 
THEOREM 2.5. Let $\mu$ be a finite measure on $\left(E_{\Delta}, \mathscr{E}_{\Delta}\right)$ and let $Y$ be in $b \tilde{\mathscr{F}}_{t}$ and $Z$ be in $b \mathscr{F}$. Then

$$
E^{\mu}\left(Y Z \circ \hat{\theta}_{t} \mid \hat{\mathscr{F}}\right)=E^{\mu}(Y \mid \hat{\mathscr{F}}) E^{\hat{X}_{0}}(Z \mid \hat{\mathscr{F}}) \circ \hat{\theta}_{t}
$$

a.s. $P^{\mu}$ for all $t$.

This theorem has several immediate corollaries.

Corollary 2.6. Let $\mu$ and $Z$ be as in 2.5. Then

$$
E^{\mu}\left(Z \circ \hat{\theta}_{t} \mid \hat{\mathscr{F}}\right)=E^{X_{0}}(Z \mid \hat{\mathscr{F}}) \circ \hat{\theta}_{t} \text { a.s. } P^{\mu} \text { for all } t \text {. }
$$

If we take $\mu=\epsilon_{x}$ and if we apply Lemma 2.4, we get the following results.

COROLlary 2.7. Let $Y$ be in $b \tilde{\mathscr{F}}_{t}$ and $Z$ be in $b \mathscr{F}_{\text {. }}$ Then, for all $t$, almost surely

$$
E^{\hat{X}_{o}}\left(Y Z \circ \hat{\theta}_{t} \mid \hat{\mathscr{F}}\right)=E^{\hat{X}_{o}}(Y \mid \hat{\mathscr{F}}) E^{\hat{X}_{o}}(Z \mid \hat{\mathscr{F}}) \circ \hat{\theta}_{t}
$$

In particular, if we set $Y \equiv 1$,

$$
E^{\hat{X}_{0}}\left(Z \circ \hat{\theta}_{t} \mid \hat{\mathscr{F}}\right)=E^{\hat{X}_{0}}(Z \mid \hat{\mathscr{F}}) \circ \hat{\theta}_{t} \quad \text { a.s. for all } t \text {. }
$$

Proof. Let us consider the following random variable

$$
W=\prod_{l}^{n} f_{i}\left(\hat{X}_{t_{i}}\right) \text {, }
$$

where $f_{i}$ are in $b \mathscr{E}_{\Delta}$ for $1 \leqq i \leqq n$ and $0 \leqq t_{1}<t_{2}<\cdots<t_{n}$. Clearly we can write $W=W_{1} W_{2} \circ \hat{\theta}_{t}$ a.s. where $W_{1}$ is in $\hat{\mathscr{F}}_{t}^{0}$ and $W_{2}$ is in $\hat{\mathscr{F}}^{0}$. Now

$$
E^{\mu}\left(W Y Z \circ \hat{\theta}_{t}\right)=E^{\mu}\left[W_{1} Y E^{\chi_{t}}\left(W_{2} Z\right)\right] .
$$

We know that $\hat{X}_{t}$ is in $F$ almost surely on $\{t<\hat{\xi}\}$. On $\{t \geqq \hat{\xi}\} \tau_{t}=\infty$ and consequently $\hat{X}_{t}=\Delta$. On the other hand, we already saw that $\hat{X}_{0}=x$ a.s. $P^{x}$ for all $x$ in $F \cup\{\Delta\}$. Therefore for all $x$ in $F \cup\{\Delta\}$,

$$
E^{x}\left(\left[W_{2} E^{\hat{x}_{0}}(Z \mid \hat{\mathscr{F}})\right]=E^{x}\left[W_{2} E^{x}(Z \mid \hat{\mathscr{F}})\right]=E^{x}\left(W_{2} Z\right) .\right.
$$

So, we have

$$
\begin{aligned}
E^{\mu}\left(W Y Z \circ \hat{\theta}_{t}\right) & =E^{\mu}\left[W_{1} Y E^{\hat{X}_{t}}\left(W_{2} E^{\hat{X}_{0}}(Z \mid \hat{\mathscr{F}})\right)\right] \\
& =E^{\mu}\left[W Y E^{\hat{X}_{0}}(Z \mid \hat{\mathscr{F}}) \circ \hat{\theta}_{t}\right] \\
& =E^{\mu}\left[W E^{\mu}(Y \mid \hat{\mathscr{F}}) E^{\hat{X}_{0}}(Z \mid \hat{\mathscr{F}}) \circ \hat{\theta}_{t}\right],
\end{aligned}
$$


if we recall that by convention $E^{\hat{X}_{0}}(Z \mid \hat{\mathscr{F}})$ is in $\hat{\mathscr{F}}$, and $\hat{\theta}_{t}$ is in $\hat{\mathscr{F}} \mid \hat{\mathscr{F}}$. Using the monotone class theorem, we see that the last equality is true for all $W$ in $b \hat{\mathscr{F}}^{0}$. If $W$ is in $b \hat{\mathscr{F}}$ there exists $W_{\mu}$ in $b \hat{\mathscr{F}}^{0}$ such that $W=W_{\mu}$ a.s. $P^{\mu}$. Hence the equality holds for every $W$ in $b \hat{F}$ and the theorem is proven. Using the corollary 2.6 , we see that the formula of the Theorem 2.5 may be written

$$
E^{\mu}\left(Y Z \circ \hat{\theta}_{t} \mid \hat{\mathscr{F}}\right)=E^{\mu}(Y \mid \hat{\mathscr{F}}) E^{\mu}\left(Z \circ \hat{\theta}_{t} \mid \hat{\mathscr{F}}\right) \text { a.s. } P^{\mu} \text { for all } t
$$

The intuitive meaning of the Theorem 2.5 , is now clear. What happened before and after the time $\tau_{t}$, are conditionally independent given the process in the support of the continuous additive functional.

We will end this section by a proposition which is closely related to Theorem 2.5.

Proposition 2.8. Let $\mu$ be a finite measure on $\left(E_{\Delta}, \mathscr{E}_{\Delta}\right)$ and let $Y$ be in $b \tilde{\mathscr{F}}_{t}$. Then

$$
E^{\mu}(Y \mid \hat{\mathscr{F}})=E^{\mu}\left(Y \mid \hat{\mathscr{F}}_{t}\right) \text { a.s. } P^{\mu}
$$

Proof. Let us prove first that for all $Z$ in $b \hat{\mathscr{F}}$,

$$
E^{\mu}\left(Z \mid \tilde{\mathscr{F}}_{t}\right)=E^{\mu}\left(Z \mid \hat{\mathscr{F}}_{t}\right) \text { a.s. } P^{\mu}
$$

If we consider $Z=\prod_{1}^{n} f_{i}\left(\hat{X}_{t_{i}}\right)$ where $f_{i}$ are in $b \mathscr{E}_{\Delta}$ and $0 \leqq t_{1}<t_{2}<\cdots<t_{n}$, then as before, we can write $Z=Z_{1} Z_{2} \circ \hat{\theta}_{t}$ a.s. where $Z_{1}$ is in $b \hat{\mathscr{F}}_{t}^{0}$ and $Z_{2}$ is in $b \hat{\mathscr{F}}^{0}$. Hence

$$
E^{\mu}\left(Z \mid \tilde{\mathscr{F}}_{t}\right)=Z_{1} E^{X_{t}} Z_{2} \quad \text { a.s. } \quad P^{\mu},
$$

and since the right hand side is in $\hat{\mathscr{F}}_{t},(2.8)$ holds. By the monotone class theorem and the properties of the completion, $(2.8)$ is clearly true for all $Z$ in $b \hat{\mathscr{F}}$.

Now, for $Z$ in $b \hat{\mathscr{F}}$ and $Y$ in $b \tilde{\mathscr{F}}_{t}$

$$
\begin{aligned}
E^{\mu}(Y Z) & =E^{\mu}\left[Y E^{\mu}\left(Z \mid \tilde{\mathscr{F}}_{t}\right)\right] \\
& =E^{\mu}\left[Y E^{\mu}\left(Z \mid \hat{\mathscr{F}}_{t}\right)\right] \\
& =E^{\mu}\left[Z E^{\mu}\left(Y \mid \hat{\mathscr{F}}_{t}\right)\right] .
\end{aligned}
$$

Hence $E^{\mu}(Y \mid \hat{\mathscr{F}})=E^{\mu}\left(Y \mid \hat{\mathscr{F}}_{t}\right)$ a.s. $P^{\mu}$. 
If we use Lemma 2.4 , this proposition has a straightforward corollary.

CoROLlaRY 2.9. Let $Y$ be in $b \tilde{\mathscr{F}}_{t} \quad$ Then $E^{\hat{x}_{0}}(Y \mid \hat{\mathscr{F}})$ is in $\hat{\mathscr{F}}_{t}$

In the following chapter we will be mainly concerned with the operator $T: b \mathscr{F} \rightarrow b \hat{\mathscr{F}}$ defined by

$$
T Z=E^{\mathrm{X}_{0}}(Z \mid \hat{\mathscr{F}})
$$

Even if this operator is not a conditional expectation, it has all its important properties. For instance

$$
\begin{aligned}
& T(\alpha Z)=\alpha T Z . \\
& T\left(Z_{1}+Z_{2}\right)=T Z_{1}+T Z_{2} . \\
& T Z \geqq 0 \quad \text { if } Z \geqq 0 . \\
& T Z_{n} \uparrow T Z \text { if } Z_{n} \uparrow Z .
\end{aligned}
$$

(2.21) If $Y$ is in $b \hat{\mathscr{F}}$ and $Z$ is in $b \mathscr{F}$ then $T(Y Z)=Y T Z$. In particular, $Y$ is in $b \hat{F}$ if an only if $T Y=Y$.

$$
T 1=1
$$

all these statements being true almost surely. They are easy to verify by applying Lemma 2.4 to the corresponding properties of the conditional expectations with respect to the measures $P^{x}$. For instance, if $Z \geqq 0$, let $B=\left\{(x, \omega): E^{x}[Z \mid \hat{\mathscr{F}}](\omega) \geqq 0\right\}$. Then $1_{B}$ is in $b \mathscr{E}_{\Delta}^{*} \otimes \hat{\mathscr{F}}$ and $1_{B}(x, \cdot)=1$ a.s. $P^{x}$ for all $x$ in $E_{\Delta}$. So $1_{B}\left[\hat{X}_{0}(\omega), \omega\right]=1$ a.s. Hence $T Z \geqq 0$ a.s. Also if $Z_{n}$ increases to $Z$,

$$
\sup _{n} E^{x}\left(Z_{n} \mid \hat{\mathscr{F}}\right)=E^{x}(Z \mid \hat{\mathscr{F}}) \text { a.s. } P^{x}
$$

for all $x$ in $E_{\Delta}$. Hence $\sup _{n} T Z_{n}=T Z$ a.s.

It is also useful to remark that if $Z=0$ a.s. $P^{x}$ for all $x$ in $F \cup\{\Delta\}$, then $T Z=0$ a.s.

Moreover the main theorem of this section and its corollaries may be expressed in terms of $T$ by the following statement: 
Let $Y$ be in $b \tilde{\mathscr{F}}_{t}$ and $Z$ be in $b \mathscr{F}$ then $T Y$ is in $b \hat{\mathscr{F}}_{t}$,

$$
T\left(Y Z \circ \hat{\theta}_{t}\right)=(T Y)(T Z) \circ \hat{\theta}_{t} \text { a.s. }
$$

and in particular

$$
T\left(Z \circ \hat{\theta}_{t}\right)=(T Z) \circ \hat{\theta}_{t} \text { a.s. }
$$

The aim of the next chapter will be to prove that under certain conditions $T$ may be considered as an integral operator.

III. The regularization. In this section, we will suppose that $X$ is a standard process with the property that the measurable space $\left(\Omega, \mathscr{F}^{0}\right)$ is a standard Borel space. This is the case if the process $X$ is of function space type, i.e., if $\Omega$ consists of all the functions from $[0, \infty]$ into $E_{\Delta}$ which are right continuous on $[0, \infty)$ and have left-hand limits on $[0, \infty)$ and if the random variables $X_{t}$ are the coordinate functions $\left[X_{t}(\omega)=\omega(t)\right]$. In this situation $\left(\Omega, \mathscr{F}^{0}\right)$ is a Polish space and then a standard Borel space. See for instance [6] and [7].

Under this hypothesis, we can prove the following theorem.

THEOREM 3.1. There exists a function

$$
P^{\omega}(A): \Omega X_{\mathscr{F}^{0}} \rightarrow R
$$

such that, for each $\omega$ in $\Omega, P^{\omega}(\cdot)$ is a probability measure on $\mathscr{F}^{0}$, for each set $A$ in $\mathscr{F}^{0}, P \cdot(A)$ is in $b \hat{\mathscr{F}}$ and for all $Z$ in $b \mathscr{F}^{0}$, the following relation holds almost surely in $\omega$.

$$
\begin{aligned}
T Z(\omega) & =E^{\hat{X}_{0}(\omega)}[Z \mid \hat{\mathscr{F}}](\omega) \\
& =E^{\omega} Z=\int_{\Omega} Z\left(\omega^{\prime}\right) P^{\omega}\left(d \omega^{\prime}\right) .
\end{aligned}
$$

Proof. Let $Q$ be the rational numbers. Since $\left(\Omega, \mathscr{F}^{0}\right)$ is a standard Borel space, there exists an increasing right continuous sequence of sets in $\mathscr{F}^{0}, A_{r}, r$ in $Q$, such that

$$
\bigcap_{r \in Q} A_{r}=\phi \text { and } \bigcup_{r \in Q} A_{r}=\Omega
$$

$\mathscr{F}^{0}$ is the $\sigma$-algebra generated by this collection. Moreover, if $F$ is a probability distribution function on this sequence, i.e., an increasing right continuous set function on this sequence with the property that 
$\inf _{r \in Q} F\left(A_{r}\right)=0$ and $\sup _{r \in Q} F\left(A_{r}\right)=1$, then $F$ can be extended in a unique way to a probability measure on $\mathscr{F}^{0}$. Indeed, this statement becomes evident if we take $A_{r}=\varphi^{-1}[(-\infty, r]]$ where $\varphi$ is a bijective measurable function from $\Omega$ into a Borel subset of the real line such that $\varphi^{-1}$ is measurable, and such a function exists by the definition of a standard Borel space.

Now let $Q^{\omega}\left(A_{r}\right)$ be versions of $T 1_{A_{r}}(\omega)$. Let us define

$$
\begin{aligned}
& N_{1}=\bigcup_{r \in Q}\left\{\omega: \inf _{\substack{s>r \\
s \in Q}} Q^{\omega}\left(A_{s}\right) \neq Q^{\omega}\left(A_{r}\right)\right\} \\
& N_{2}=\left\{\omega: \sup _{r \in Q} Q^{\omega}(A r) \neq 1\right\} \\
& N_{3}=\left\{\omega: \inf _{r \in Q} Q^{\omega}\left(A_{r}\right) \neq 0\right\} \\
& N=N_{1} \cup N_{2} \cup N_{3} .
\end{aligned}
$$

Clearly, $N$ is in $\hat{\mathscr{F}}$. Moreover for all finite measures $\mu$ on $\left(E_{\Delta}, \mathscr{E}_{\Delta}\right)$, $P^{\mu}(N)=0$. Indeed, $T 1_{A_{r}} \leqq \inf _{s>r} T 1_{A_{s}}$ a.s. and if $s_{n}$ decreases to $r, 1_{A_{s n}}$ decreases to $1_{A r}$ and consequently $T 1_{A_{s n}}$ decreases to $T 1_{A r}$ a.s. This implies that $T 1_{A r}=\inf _{s>r} T 1_{A_{s}}$ a.s. Similarly $\sup _{r \in Q} T 1_{A_{r}}=1$ a.s. and $\inf _{r \in Q} T 1_{A_{r}}=o$ a.s.

Now let $F$ be any probability distribution function on the sequence $\left\{A_{r}: r\right.$ in $\left.Q\right\}$ and let us define

$$
P^{\omega}\left(A_{r}\right)=Q^{\omega}\left(A_{r}\right) 1_{N^{c}}(\omega)+F\left(A_{r}\right) 1_{N}(\omega) .
$$

clearly $P \cdot\left(A_{r}\right)$ is in $b \hat{\mathscr{F}}$ for all $r$ in $Q$ and for all $\omega$ in $\Omega P^{\omega}$ is a probability distribution function on the sequence $A_{r}$. Let us also denote by $P^{\omega}$, the unique extension of $P^{\omega}$ to a probability measure on $\mathscr{F}^{0}$. If we define $\mathscr{C}=\left\{A \in \mathscr{F}^{0}: P \cdot(A) \in \hat{\mathscr{F}}\right\}$ then $\mathscr{C}$ is a $\sigma$-algebra containing $A_{r}$ for all $r$ in $Q$. Hence for all $A$ in $\mathscr{F}^{0}, P \cdot(A)$ is in $\hat{\mathscr{F}}$. Now let $H=\left\{Z \in b \mathscr{F}^{0}: T Z=E \cdot Z\right.$ a.s. $\} . \quad H$ is a linear space containing $1_{A_{r}}$ for all $r$ in $Q$. Moreover if $Z_{n}$ in $H^{+}$increases to $Z$ bounded, then $Z$ is in $H$. By the monotone class theorem, the proof is complete if we remark that the collection $\left\{A_{r}-A_{s}: s<r, s, r \in Q\right\}$ is a $\pi$-system generating $\mathscr{F}^{0}$.

From now on, we will restrict our attention to the stochastic process $\tau=\left\{\tau_{t}: 0 \leqq t<\infty\right\}$. Unhappily, the measures $P^{\omega}$ we have just constructed can only be defined on $\mathscr{F}^{0}$ and in the general case, $\tau$ is not $\mathscr{F}^{0}$ measurable. However, if we suppose that there exists a reference 
measure for $X$ (see [1] V-1), then the C.A.F. $A$ is equivalent to a perfect C.A.F. $B$ such that each $B_{t}$ is in $\mathscr{F}^{0}$ (see [1] V-2.1 and 2.10). So without loss of generality, we may and we will assume that $A$ is a perfect C.A.F. and each $A_{t}$ is $\mathscr{F}^{0}$ measurable.

Since $\left\{\tau_{t}<s\right\}=\left\{A_{s}>t\right\}$, that implies that the process $\tau$ is $\mathscr{F}^{0}$ measurable. Moreover in this situation the support of the C.A.F. is a Borel set for $P^{x}\left(\tau_{0}=0\right)$ is in $b \mathscr{E}_{\Delta}$.

One 1..ore remark: Later we will have to consider the increments of the process $\tau$, i.e., $\tau_{t+s}-\tau_{t}$, and this is not defined on the set $\left\{\tau_{t}=\infty\right\}$. However if we set $\tau_{t+s}-\tau_{t}=\infty$ on $\left\{\tau_{t}=\infty\right\}$, this random variable is in $\mathscr{F}^{0}$ and $\tau_{t+s}-\tau_{t}=\tau_{s} \circ \hat{\theta}_{t}$ a.s. Indeed

$$
\begin{aligned}
P^{x}\left[\tau_{s} \circ \hat{\theta}_{t}<\infty, \tau_{t}=\infty\right] & =E^{x}\left[P^{X_{t}}\left(\tau_{s}<\infty\right) ; \tau_{t}=\infty\right] \\
& =P^{\Delta}\left(\tau_{s}<\infty\right) P^{x}\left(\tau_{t}=\infty\right)=0
\end{aligned}
$$

for $\tau_{0}=\infty$ a.s. $P^{\Delta}$.

We are now ready to state the main theorem of this section.

THEOREM 3.2. There exists a set $N$ in $\hat{F}$ with $P^{\mu}(N)=0$ for all finite measures $\mu$ on $\left(E_{\Delta}, \mathscr{E}_{\Delta}\right)$, such that for all $\omega$ in $\{\hat{\xi}>0\}-N$, the process

$$
\left\{\tau_{t}: 0 \leqq t<\hat{\xi}(\omega)\right\}
$$

is an additive process on $\left(\Omega, \mathscr{F}^{0}, P^{\omega}\right)$ (i.e., a process with independent increments such that $\tau_{0}=0$ a.s. $\left.P^{\omega}\right)$.

Proof. Let us prove first that for all $t \geqq 0$ the $\sigma$-algebras

$$
\mathscr{K}_{t}=\sigma\left\{\tau_{s}: 0 \leqq s \leqq t\right\}
$$

and

$$
\mathscr{L}_{t}=\sigma\left\{\tau_{t+s}-\tau_{t}: 0<s<\infty\right\}
$$

are independent with respect to $P^{\omega}$ for almost all $\omega$. Indeed the right continuity of $\tau$, implies that $\mathscr{K}_{t}$ and $\mathscr{L}_{t}$ are generated by countable $\pi$-systems containing $\Omega$, let us say $\mathscr{K}_{t}^{0}$ and $\mathscr{L}_{t}^{0}$ respectively. On the other hand, $\mathscr{K}_{t}$ is contained in $\mathscr{F}_{t}$ and $\mathscr{L}_{t}$ is contained in $\hat{\theta}_{t}^{-1}(\mathscr{F})$. Using Corollary 2.7 , this implies that 


$$
P^{\omega}(A \cap B)=P^{\omega}(A) P^{\omega}(B) \text { a.s. }
$$

for $A$ in $\mathscr{K}_{t}$ and $B$ in $\mathscr{L}_{t}$.

Let us define

$$
N_{t}=\bigcup_{A \in \mathscr{K}_{t}^{0}} \bigcup_{B \in \mathscr{L}_{t}^{0}}\left\{\omega: P^{\omega}(A \cap B) \neq P^{\omega}(A) P^{\omega}(B)\right\}
$$

Clearly $N_{t}$ is in $\hat{\mathscr{F}}$ and $P^{\mu}\left(N_{t}\right)=0$ for all $\mu$. Using twice the monotone class theorem, it is easy to see that for all $\omega$ in $N_{t}^{c}$,

$$
P^{\omega}(A \cap B)=P^{\omega}(A) P^{\omega}(B)
$$

for all $A$ in $\mathscr{K}_{t}, B$ in $\mathscr{L}_{t}$.

Now let $N_{1}=\cup_{t \in Q^{+}} N_{t}$. Then for all $\omega$ in $N_{1}^{c}, t \geqq 0, s>0, \tau_{t+s}-\tau_{t}$ is independent of $\mathscr{K}_{t}$ with respect to $P^{\omega}$. For if $A$ is in $\mathscr{K}_{t}$ and $u \geqq 0$, let us choose $r_{n}$ in $Q^{+}$such that $r_{n} \downarrow t$ and $r_{n}<t+s$. Then, by the right continuity of $\tau$, we have

$$
\begin{aligned}
P^{\omega}\left[A \cap\left\{\tau_{t+s}-\tau_{t} \leqq v\right\}\right] & =\lim _{r_{n} \downarrow t} P^{\omega}\left[A \cap\left\{\tau_{t+s}-\tau_{r_{n}} \leqq v\right\}\right] \\
& =\lim _{r_{n} \downarrow t} P^{\omega}(A) P^{\omega}\left(\tau_{t+s}-\tau_{r_{n}} \leqq v\right) \\
& =P^{\omega}(A) P^{\omega}\left(\tau_{t+s}-\tau_{t} \leqq v\right) .
\end{aligned}
$$

Now since $\{\hat{\xi} \leqq t\}=\left\{\hat{X}_{t}=\Delta\right\}, \hat{\xi}$ is in $\hat{F}$ and so

$$
P^{\omega}[\hat{\xi} \neq \hat{\xi}(\omega)]=0
$$

for almost all $\omega$. Also $e^{-\tau_{0}}=1_{F}\left(\hat{X}_{0}\right)$ a.s. $P^{x}$ for all $x$ in $F \cup\{\Delta\}$. Hence by Lemma 2.4

$$
E^{\omega}\left(e^{-\tau_{0}}\right)=1_{F}\left[\hat{X}_{0}(\omega)\right]
$$

for almost all $\omega$. Let $N_{2}$ be the set of $\omega$ 's for which either (3.2) or (3.3) is not satisfied and let $N=N_{1} \cup N_{2}$. Clearly $N$ is in $\hat{F}$ and $P^{\mu}(N)=0$ for all $\mu$. If $\omega$ is in $\{\hat{\xi}>0\}-N, \tau_{0}=0$ a.s. $P^{\omega}$ and the process $\tau$ is finite a.s. $P^{\omega}$ on $[0, \xi(\omega))$ since $\hat{\xi}=\hat{\xi}(\omega)$ a.s. $P^{\omega}$. Also for all $t<\hat{\xi}(\omega)$ and $s$ in $(0, \hat{\xi}(\omega)-t) \tau_{t+s}-\tau_{t}$ is independent of $\sigma\left\{\tau_{\cup}: 0 \leqq U \leqq t\right\}$ with respect to $P^{\omega}$. That concludes the proof of the theorem. 
Let us remark that there is no interest in considering the process $\tau$ with respect to $P^{\omega}$ for $\omega$ in $\{\hat{\xi}=0\}$ because (3.3) implies that $\tau_{0}=\infty$ a.s. $P^{\omega}$ for almost all $\omega$ in $\{\hat{\xi}=0\}$.

Let us also note that the process $\tau$ is not homogeneous. Indeed

$$
P^{\omega}\left(\tau_{t+s}-\tau_{t} \in B\right)=P^{\hat{\theta}_{t}(\omega)}\left(\tau_{s} \in B\right)
$$

for almost all $\omega$ in $\Omega$.

If we consider the particular case where the support of the C.A.F. is a single point $x_{0}$, then

$$
\hat{\mathscr{F}}^{0}=\sigma\left(A_{\infty}\right)
$$

for $\left\{\hat{X}_{t}=x_{0}\right\}=\left\{A_{\infty}>t\right\}$. Moreover there exists $\gamma \geqq 0$, such that $P^{x_{0}}\left(A_{\infty}>t\right)=e^{-\gamma t}$. If $\gamma=0, A_{\infty}=\infty$ a.s. $P^{x_{0}}$ and it follows easily that $P^{\omega}=P^{x_{0}}$ for almost all $\omega$ in $\Omega$. In this situation, we have as a corollary of Theorem 3.2, that the process $\tau=\left\{\tau_{t}: 0 \leqq t<\infty\right\}$ is a homogeneous additive process with increasing paths on $\left(\Omega, \mathscr{F}^{0}, P^{x_{0}}\right)$.

It is now clear that Theorem 3.2 generalizes the theorem which appears in [1]-V-3.21.

In order to obtain a Levy's decomposition of the process $\tau$, Theorem 3.2 is not sufficient. We also need the fact that the process $\tau$ is continuous in probability with respect to $P^{\omega}$ for almost all $\omega$ in $\{\hat{\xi}>0\}$ or equivalently the functions $t \rightarrow E^{\omega}\left(e^{-\tau_{t}}\right)$ are continuous on $[0, \hat{\xi}(\omega))$ for almost all $\omega$ in $\{\hat{\xi}>0\}$. Indeed, since $E^{\omega}\left(e^{-\tau_{t}}\right)=$ $E^{\omega}\left(e^{-\tau_{t-s}}\right) E^{\omega}\left(e^{-\left(\tau_{t}-\tau_{t-s}\right)}\right)$ if we let $s$ decrease to zero, we have $E^{\omega}\left(e^{-\tau_{t}}\right)=$ $E^{\omega}\left(e^{\left.-\tau_{t-}\right)} E^{\omega}\left[e^{-\left(\tau_{t}-\tau_{t-}\right)}\right]\right.$ and then if $t<\hat{\xi}(\omega) \tau_{t-}=\tau_{t}$ a.s. $P^{\omega}$ if and only if $E^{\omega}\left(e^{-\tau_{t}}\right)=E^{\omega}\left(e^{-\tau_{t}-}\right)$.

It is easy to see that in the general case, this condition will not be satisfied. However we have the following theorem.

Theorem 3.3. There exists $N$ in $\hat{\mathscr{F}}$ with $P^{\mu}(N)=0$ for all finite measures $\mu$ on $\left(E_{\Delta}, \mathscr{E}_{\Delta}\right)$, such that for all $\omega$ in $\{\hat{\xi}>0\}-N$, the function $t \rightarrow E^{\omega}\left(e^{-\tau_{t}}\right)$ is continuous on $[0, \hat{\xi}(\omega))$ if and only if the following assumption holds.

Assumption 3.4. For all $\left\{\hat{\mathscr{F}}_{t}\right\}$ stopping times $T$,

$$
\tau_{T-}=\tau_{T} \text { a.s. on }\{0<T<\hat{\xi}\}
$$

Proof. Let $\Omega_{0}=\Omega-N$ where $N$ is the set of measure zero appearing in the statement of Theorem 3.2. Let us set $C_{t}(\omega)=e^{-\tau_{t}(\omega)}$ and $\hat{C}_{t}(\omega)=E^{\omega}\left(e^{-\tau_{t}}\right)$ if $\omega$ is in $\Omega_{0}$ and $\hat{C}_{t}(\omega)=0$ otherwise. Then we define for $\epsilon>0$, 


$$
T_{\epsilon}=\inf \left\{t>0: \hat{C}_{t-}-\hat{C}_{t} \geqq \epsilon\right\}
$$

and $T_{\epsilon}=\hat{\xi}$ if the set in braces is empty. Now if we prove that for all finite measures $\mu$ on $\left(E_{\Delta}, \mathscr{C}_{\Delta}\right), P^{\mu}\left(T_{\epsilon}<\hat{\xi}\right)=0$, the sufficiency is established. Indeed if $N^{\prime}=\cup_{n}\left\{T_{n}^{1}<\hat{\xi}\right\}, P^{\mu}\left(N^{\prime}\right)=0$ and for all $\omega$ in $\{\hat{\xi}>0\}-N^{\prime}, \hat{C}_{t-}(\omega)=\hat{C}_{t}(\omega)$ for all $t<\hat{\xi}(\omega)$. This implies the continuity of $E^{\omega}\left(e^{-\tau_{t}}\right)$ on $[0, \hat{\xi}(\omega)]$ for all $\omega$ in $\{\hat{\xi}>0\}-\left(N \cup N^{\prime}\right)$.

Let us write $T_{\epsilon}=T$. Since $\hat{C}_{t}=0$ on $\{\hat{\xi} \leqq t\}, T \leqq \hat{\xi}$. Moreover $T$ is a $\left\{\hat{\mathscr{F}}_{t}\right\}$ stopping time for $\{T \leqq t\}=\{T \leqq t, \hat{\xi}>t\} \cup\{\hat{\xi} \leqq t\}$ and if $Q_{t}$ denotes the rationals in $(0, t)$

$$
\begin{gathered}
\{T \leqq t<\hat{\xi}\}=\bigcap_{m} \bigcup_{\substack{r \in Q_{t} \backslash\{t\} \\
s \in Q_{t}}}\left\{\hat{C}_{s}-\hat{C}_{r} \geqq \epsilon, \hat{\xi}>t\right\} \\
0<r-s<\frac{1}{m} .
\end{gathered}
$$

Hence $\{T \leqq t\}$ is in $\hat{\mathscr{F}}_{t}$ since $\hat{C}_{t}$ is clearly in $\hat{\mathscr{F}}_{t \cdot}$ Also if $T(\omega)<\hat{\xi}(\omega)$, $\hat{C}_{T(\omega)-}(\omega)-\hat{C}_{T(\omega)}(\omega) \geqq \epsilon$.

Now if $G\left(\omega, \omega^{\prime}\right)$ is in $b \hat{F} \otimes \mathscr{F}^{0}$ and if $\bar{G}(\omega)=G(\omega, \omega)$ it is easy to see that for almost all $\omega$,

$$
E^{X_{0}(\omega)}[\bar{G} \mid \mathscr{F}](\omega)=\int_{\Omega} G\left(\omega, \omega^{\prime}\right) P^{\omega}\left(d \omega^{\prime}\right)
$$

Since for all $\left\{\hat{\mathscr{F}}_{t}\right\}$ stopping times $T, \tau_{T(\omega)}\left(\omega^{\prime}\right)$ and $\tau_{T(\omega)-}\left(\omega^{\prime}\right)$ are clearly in $\hat{\mathscr{F}} \otimes \mathscr{F}^{0}$, we have

$$
\begin{aligned}
& \hat{C}_{T}=E^{\mathrm{X}_{0}}\left(C_{T} \mid \hat{F}\right) \quad \text { a.s. } \quad \text { and } \\
& \hat{C}_{T^{-}}=E^{\chi_{0}}\left(C_{T-} \mid \hat{F}\right) \quad \text { a.s. }
\end{aligned}
$$

Hence

$$
\begin{aligned}
P^{\mu}(T<\hat{\xi}) & \leqq \frac{1}{\epsilon} E^{\mu}\left[\hat{C}_{T-}-\hat{C}_{T} ; T<\hat{\xi}\right] \\
& \leqq \frac{1}{\epsilon} E^{\mu}\left\{E^{x_{0}}\left[\left(C_{T-}-C_{T}\right) 1_{\{T<\hat{\xi}\}} \mid \hat{F}\right] ; \hat{\xi}>0\right\} \\
& \leqq \frac{1}{\epsilon} E^{\mu}\left\{E^{x_{0}}\left[\left(C_{T-}-C_{T}\right) 1_{\{T<\hat{\xi}\}} \mid \hat{F}\right] \circ \hat{\theta}_{0} ; \hat{\xi}>0\right\} \\
& \leqq \frac{1}{\epsilon} E^{\mu}\left\{E^{x_{0}}\left[C_{T-}-C_{T} ; T<\hat{\xi}\right] ; \hat{\xi}>0\right\} \\
& \leqq 0
\end{aligned}
$$

for $C_{T-}=C_{T}$ a.s. on $\{0<T<\hat{\xi}\}$. 
For the necessity of Assumption 3.4, note that for all $\left\{\hat{\mathscr{F}}_{t}\right\}$ stopping times $T, \hat{C}_{T-}=\hat{C}_{T}$ a.s. on $\{0<T<\hat{\xi}\}$. Then for all $x$ in $F$,

$$
\begin{aligned}
0 & =E^{x}\left[\hat{C}_{T-}-\hat{C}_{T} ; 0<T<\hat{\xi}\right] \\
& =E^{x}\left[E^{x_{0}}\left(C_{T-}-C_{T} \mid \hat{\mathscr{F}}\right) ; 0<T<\hat{\xi}\right] \\
& =E^{x}\left[C_{T-}-C_{T} ; 0<T<\hat{\xi}\right]
\end{aligned}
$$

and so $\tau_{T-}=\tau_{T}$ a.s. $P^{x}$ on $\{0<T<\hat{\xi}\}$ for all $x$ in $F$. Now since $\tau_{t}=\tau_{0}+\tau_{t} \circ \theta_{\tau_{0}}$ for all $t$ almost surely, it is clear that $\tau_{t-}=\tau_{0}+\tau_{t-} \circ \theta_{\tau_{0}}$ for all $t$ almost surely and then $\tau_{T}-\tau_{T-}=\left(\tau_{T}-\tau_{T-}\right) \circ \hat{\theta}_{0}$ a.s. on $\{0<T<\hat{\xi}\}$. Hence for all finite measures $\mu \quad$ on $\quad\left(E_{\Delta}, \mathscr{E}_{\Delta}\right), P^{\mu}\left(\tau_{T}-\tau_{T-}=0, \quad 0<T<\hat{\xi}\right)=P^{\mu}\left[\left(\tau_{T}-\tau_{T-}\right) \circ \hat{\theta}_{0}=0\right.$, $\left.0<T \circ \hat{\theta}_{0}<\hat{\xi} \circ \hat{\theta}_{0}, \hat{\xi}>0\right]=E^{\mu}\left[P^{x_{0}}\left(\tau_{T}-\tau_{T-}=0,0<T<\hat{\xi}\right) ; \hat{\xi}>0\right]=0$ since $\hat{X}_{0}$ is in $F$ on $\{\hat{\xi}>0\}$.

This finishes the proof of Theorem 3.3 and we have this straightforward corollary.

COROLlaRY 3.5. Under the Assumption 3.4, there exists $N$ in $\hat{F}$ with $P^{\mu}(N)=0$ for all finite measures $\mu$ on $\left(E_{\Delta}, \mathscr{E}_{\Delta}\right)$, such that for all $\omega$ in $\{\hat{\xi}>0\}-N$, the process $\left\{\tau_{t}: 0 \leqq t<\hat{\xi}(\omega)\right\}$ is a Levy process with increasing paths on $\left(\Omega, \mathscr{F}^{0}, P^{\omega}\right)$.

IV. The decomposition. Since $\tau$ is an increasing right continuous process, we can decompose it into its continuous and purely discontinuous parts. Let

$$
\tau_{t}=\tau_{0}+\tau_{t}^{c}+\tau_{t}^{j}
$$

be this decomposition. If we denote by $K(\omega)$ the set of discontinuity points of the function $t \rightarrow \tau_{t}(\omega)$ for $t>0$, then

$$
\begin{gathered}
\tau_{t}^{c}=\int_{(0, t]} 1_{K^{c}}(s) d \tau_{s} . \\
\tau_{t}^{j}=\sum_{0<s \leqq t}\left(\tau_{s}-\tau_{s-}\right) .
\end{gathered}
$$

Let us first restrict our attention to the continuous part of $\tau$.

Lemma 4.1. Let $\tau_{t}^{c}$ be the continuous part of $\tau_{t}$. Then, almost surely,

$$
\tau_{t}^{c}=\int_{0}^{\tau_{t}} 1_{F}\left(X_{s}\right) d s \text { for all } t
$$


Proof. Let us recall the following change of variables formula. If $a(t)$ is a nonnegative increasing right continuous function on $[0, \infty]$ and

$$
\bar{a}(t)=\inf \{s: a(s)>t\}
$$

then for all nonnegative Borel functions $g$ on $[0, \infty)$

$$
\int_{(0, \infty)} g(t) d a(t)=\int_{a(0)}^{a(\infty)} g[\bar{a}(t)] d t,
$$

where $a\left((\infty)=\lim _{t \uparrow \infty} a(t)\right.$. Applying this formula to (4.2), we have

$$
\tau_{t}^{c}=\int_{\tau_{0}}^{\tau_{\infty}} 1_{(0, t]}\left(A_{s}\right) 1_{K^{c}}\left(A_{s}\right) d s
$$

It is easy to see that

$$
1_{(0, t)}\left(A_{s}\right)=1_{\left(\pi, \tau_{t}\right)}(s)
$$

Moreover $A_{s}$ is in $K$ if and only if $s$ is in $R^{c} \cup L^{c}$, where $R(L)$ denotes the set of points of right (left) increase of $A$. Indeed, since $\tau_{A_{s^{-}}} \leqq s \leqq$ $\tau_{A_{s}}$, if $A_{s}$ is in $K$ there exists $v \neq s$ such that $\tau_{A_{s}-}<v<\tau_{A_{s}}$. But then

$$
A_{v}=\inf \left\{u: \tau_{u}>v\right\}=A_{s} .
$$

On the other hand, if $A_{v}=A_{s}$ for $v \neq s, \tau_{A_{s}} \leqq v \wedge s$ and $\tau_{A_{s}} \geqq$ $v \vee s$. Hence $A_{s}$ is in $K$.

But we also know that almost surely

$$
R \subset\left\{s: X_{s} \in F\right\} \subset R \cup L
$$

(see [1]-V-3.8). Moreover $R \cup L-R \cap L$ is a countable set. Therefore $1_{K^{c}}\left(A_{s}\right)=1_{F}\left(X_{s}\right)$ for almost all $s$, and

$$
\tau_{t}^{c}=\int_{\tau_{0}}^{\tau_{t}} 1_{F}\left(X_{s}\right) d s \text { a.s. }
$$

Moreover, since $\tau_{0}=T_{F}$ a.s. $X_{s}$ is in $F^{c}$ for all $s<\tau_{0}$ a.s. and so the result.

Using this representation, we have the following theorem. 
THeOREM 4.2. Let $\tau^{c}$ be the continuous part of $\tau$. Then $\tau^{c}$ is a continuous additive functional of the process $\hat{X}$. In particular, $\tau_{t}^{c}$ is in $\hat{\mathscr{F}}_{t}$ for all $t$ and

$$
\tau_{t+s}^{c}=\tau_{t}^{c}+\tau_{s}^{c} \circ \hat{\theta}_{t}
$$

almost surely for all $t, s$.

Proof. It is clear that the function $t \rightarrow \tau_{t}^{c}$ is nondecreasing and continuous and $\tau_{0}^{c}=0$ almost surely. Moreover for all $t \geqq \hat{\xi}=A_{\infty}$

$$
\tau_{t}^{c}=\tau_{\xi}^{c}=\int_{0}^{\infty} 1_{F}\left(X_{s}\right) d s
$$

and $\tau_{t}^{c}=0$ for all $t$ a.s. $P^{\Delta}$.

Now, let us consider

$$
B_{t}=\int_{0}^{t} 1_{F}\left(X_{s}\right) d s
$$

$B$ is a perfect continuous additive functional of $X$ and by its strong additivity property we have

$$
\begin{aligned}
\tau_{t+s}^{c} & =B_{\tau_{t+s}} \\
& =B_{\tau_{t}+\tau_{s} \circ \hat{\theta}_{t}} \\
& =B_{\tau_{t}}+B_{\tau_{s} \circ \hat{\theta}_{t}}\left[\hat{\theta}_{t}\right] \\
& =B_{\tau_{t}}+B_{\tau_{s}} \circ \hat{\theta}_{t} \\
& =\tau_{t}^{c}+\tau_{s}^{c} \circ \hat{\theta}_{t} \text { a.s. }
\end{aligned}
$$

All that remains to be proved is the measurability of $\tau_{t}^{c}$. Let $D=\operatorname{supp} B$. Since $B_{r_{0}}=0$ a.s., $T_{D} \geqq \tau_{0}$ a.s. and

$$
D=\left\{x: P^{x}\left[T_{D}=0\right]=1\right\} \subset F .
$$

Furthermore

$$
u_{B}^{\alpha}(x)=\int_{0}^{\infty} e^{-\alpha t} P^{x}\left(X_{t} \in F\right) d t \leqq \frac{1}{\alpha}
$$

In this situation, for each finite measure $\mu$ on $\left(E_{\Delta}, \mathscr{E}_{\Delta}\right)$, there exists a 
sequence $g_{n}$ in $b_{\mathscr{E}_{\Delta}^{*+}}^{*}$ such that $B_{t}^{n}=\int_{0}^{t} g_{n}\left(X_{s}\right) d A_{s}$ converges to $B_{t}$ on $[0, \infty)$ almost surely $P^{\mu}$, the convergence being uniform on each compact subinterval (see [3]). Hence

$$
B_{\tau_{t}}^{n} 1_{\left\{\tau_{t}<\infty\right\}} \rightarrow \tau_{t}^{c} 1_{\left\{\tau_{t}<\infty\right\}} \text { a.s. } P^{\mu} .
$$

But

$$
\begin{aligned}
B_{\tau_{t}}^{n} 1_{\left\{\tau_{t}<\infty\right\}} & =\int_{0}^{\tau_{t}} g_{n}\left(X_{s}\right) d A_{s} 1_{\left\{\tau_{t}<\infty\right\}} \\
& =\int_{0}^{t} g_{n}\left(\hat{X}_{s}\right) d s 1_{\{0, \hat{\xi})}(t) .
\end{aligned}
$$

So $B_{\tau_{t}}^{n} 1_{\left\{\tau_{t}<\infty\right\}}$ is in $\hat{\mathscr{F}}_{t}$ for $\left\{\tau_{t}<\infty\right\}=\left\{\hat{X}_{t} \in F\right\}=\{t<\hat{\xi}\}$. Then $\tau_{t}^{c} 1_{[0, \hat{\xi})}(t)$ is in $\hat{\mathscr{F}}_{t}$ for all $t$. Now since $\tau_{t}^{c}$ is continuous and constant on $\{t \geqq \hat{\xi}\}$,

$$
\tau_{t}^{c}=\int_{0}^{t} 1_{[0, \hat{\xi})}(s) d \tau_{s}^{c}
$$

Hence

$$
\tau_{t}^{c}=\lim _{n \rightarrow \infty} \sum_{k=1}^{2^{n}}\left[\tau_{\left(k / 2^{n}\right) t}^{c}-\tau_{\left((k-1) / 2^{n}\right) t}^{c}\right] 1_{[0, \xi)}\left(\frac{k}{2^{n}} t\right)
$$

But

$$
\left[\tau_{\left(k / 2^{n}\right) t}^{c}-\tau_{\left((k-1) / 2^{n}\right) t}^{c}\right] 1_{[0, \hat{\xi})}\left(\frac{k}{2^{n}} t\right)
$$

is in $\hat{\mathscr{F}}_{\left(k / 2^{n}\right) t} \subseteq \hat{\mathscr{F}}_{t}$ and so $\tau_{t}^{c}$ is in $\hat{\mathscr{F}}_{t}$ for all $t$. This completes the proof of Theorem 4.2.

Let us turn now to the purely discontinuous part of the process $\tau$.

If $\mathscr{B}$ is the collection of Borel sets on $(0, \infty)$, let us define for $B$ in $\mathscr{B}$ and $t$ in $[0, \infty)$,

$$
M_{t}(B)=\left|\left\{s \in(0, t]: \tau_{s}-\tau_{s^{-}} \in B\right\}\right|,
$$

i.e., the number of points $s$ in $(0, t]$ such that $\tau_{s}-\tau_{s-}$ is in $B$. Clearly for each $\omega$ in $\Omega$ and $B$ in $\mathscr{B}$ bounded away from zero [i.e., $B \subseteq(1 / n, \infty)$ for some $n<\infty]$ the paths $t \rightarrow M_{t}(B)(\omega)$ are right continuous step functions with jumps of size 1 . Also $M_{0}(B)=0$ and $M_{\hat{\xi}}(B)=$ $M_{\infty}(B)$. Now we have the following lemma.

Lemma 4.3. For each $\omega$ in $\{\hat{\xi}>0\}$ and $t$ in $[0, \hat{\xi}(\omega)), M_{t}(B)(\omega)$ is a $\sigma$-finite measure on $\mathscr{B}$. Moreover for all positive Borel functions $g$ on $[0, \infty]$ such that $g(0)=0$ and for all $t<\hat{\xi}$, 


$$
\sum_{0<s \leqq t} g\left(\tau_{s}-\tau_{s-}\right)=\int_{(0, \infty)} g(u) M_{t}(d u) .
$$

In particular,

$$
\tau_{t}^{j}=\int_{(0, \infty)} u M_{t}(d u)
$$

Proof. Clearly $M_{t}(B)$ is a counting measure such that $M_{t}[(1 / n, \infty)]<\infty$ since $\tau_{t}<\infty$. Now if $g=1_{B}$ for $B$ in $\mathscr{B}$, both sides of the equality (4.7) are equal to $M_{t}(B)$ and so by the monotone class theorem, (4.7) holds for all positive Borel functions $g$ on $[0, \infty)$ with $g(0)=0$, the latter condition preserving the countability of the sum of the left-hand side.

From now on let us fix $\omega$ in $\{\hat{\xi}>0\}-N$, where $N$ is the set of measure zero appearing in the statement of Corollary 3.5. It follows from the proof of Theorem 3.2, that we can suppose without loss of generality that $\tau_{0} \equiv 0$ and $\hat{\xi} \equiv \hat{\xi}(\omega)$ since we are now only concerned with the measure $P^{\omega}$. From the general theory of Levy processes we have the following theorem.

THEOREM 4.4. Under the Assumption 3.4, there exists $N$ in $\hat{\mathscr{F}}$ with $P^{\mu}(N)=0$ for all finite measures $\mu$ on $\left(E_{\Delta}, \mathscr{E}_{\Delta}\right)$, such that for all $\omega$ in $\{\hat{\xi}>0\}-N$, for all sets $B$ in $\mathscr{B}$, the process $M_{t}(B), 0 \leqq t<\hat{\xi}(\omega)$, is a Levy process of Poisson type (possibly with infinite parameter) on $\left(\Omega, \mathscr{F}^{0}, P^{\omega}\right)$. In particular,

$$
E^{\omega}\left[e^{-\alpha M_{t}(B)}\right]=e^{-\left(1-e^{-\alpha}\right) E^{\omega} N_{t}(B)} .
$$

Proof. We will only sketch the proof since this result is well known (see, for instance, [5]-I where it is treated in full detail). It is not too difficult to see that $M_{t}(B)$ is measurable with respect to $\mathscr{K}_{t}=$ $\sigma\left\{\tau_{s}: 0<s \leqq t\right\}$ for all $B$ in $\mathscr{B}$ and $t<\hat{\xi}(\omega)$. And so this process has independent increments by Theorem 3.2. It is also continuous in probability since

$$
P^{\omega}\left[M_{t-}(B)<M_{t}(B)\right] \leqq P^{\omega}\left(\tau_{t-}<\tau_{t}\right)=0
$$

by Theorem 3.3.

Therefore if $B$ in $\mathscr{B}$ is bounded away from zero, by the Poisson law of rare events, there exists $\lambda<\infty$ such that

$$
E^{\omega}\left[e^{-\alpha M_{t}(B)}\right]=e^{-\lambda\left(1-e^{-\alpha}\right)} .
$$

Hence $\lambda=E^{\omega} M_{t}(B)$. 
If $B$ is arbitrary, let $B_{n}=B \cap(1 / n, \infty)$. Then $M_{t}\left(B_{n}\right)$ increases to $M_{t}(B)$ and $E^{\omega} M_{t}\left(B_{n}\right)$ increases to $E^{\omega} M_{t}(B)$. Hence

$$
E^{\omega}\left[e^{-\alpha M_{t}(B)}\right]=e^{-\left(1-e^{-\alpha}\right) E^{\omega} M_{t}(B)} .
$$

Using this result it is easy to see that if we define

$$
\nu_{t}(B)(\omega)=E^{\omega} M_{t}(B),
$$

then for all $\omega$ in $\{\hat{\xi}>0\}-N$, for all $t<\hat{\xi}(\omega), \nu_{t}(\cdot)(\omega)$ is a $\sigma$-finite measure on $\mathscr{B}$ which is finite on the sets in $\mathscr{B}$ bounded away from zero.

Moreover for each $\omega$ in $\{\hat{\xi}>0\}-N$ and for each $B$ in $\mathscr{B}$ bounded away from zero, the function $t \rightarrow \nu_{t}(B)(\omega)$ is increasing and continuous on $[0, \hat{\xi}(\omega))$.

Regrouping all the results we have about the structure of the process $\tau$, we can state the following theorem.

THEOREM 4.5. Under the Assumption 3.4, there exists $N$ in $\hat{\mathscr{F}}$ with $P^{\mu}(N)=0$ for all finite measures $\mu$ on $\left(E_{\Delta}, \mathscr{E}_{\Delta}\right)$ such that for all $\omega$ in $\{\hat{\xi}>0\}-N$, and for all $t$ in $[0, \hat{\xi}(\omega))$,

$$
\tau_{t}=\tau_{t}^{c}+\int_{(0, \infty)} u M_{t}(d u) \text { a.s. } P^{\omega}
$$

where $\tau_{t}^{c}$ is a continuous additive functional of $\hat{X}$ and $M_{t}(B)$ is a Levy process of Poisson type for each set $B$ in $\mathscr{B}$.

Moreover if $\nu_{t}(B)(\omega)=E^{\omega} M_{t}(B)$, then $\nu_{t}(\cdot)(\omega)$ is a Levy measure and

$$
E^{\omega}\left(e^{-\alpha \tau_{t}}\right)=\exp \left[-\alpha \tau_{t}^{c}(\omega)-\int_{(0, \infty)}\left(1-e^{-\alpha u}\right) \nu_{t}(d u)(\omega)\right]
$$

Proof. All we have to prove is (4.13). Since $\tau_{t}^{c}$ is $\hat{\mathscr{F}}_{t}$ measurable, we have

$$
E^{\omega}\left(e^{-\alpha \tau_{t}}\right)=e^{-\alpha \tau_{f}^{c}(\omega)} E^{\omega}\left[e^{-\alpha \int_{(0,0)} u M_{t}(d u)}\right] \text { a.s. }
$$

Since both sides of the equality are continuous in $t$ and $\alpha$, by subtracting another set of measure zero, the equality holds for all $t$ and $\alpha$ almost surely in $\omega$.

Now it follows from the general theory of Levy processes that for $B_{k}, 1 \leqq k \leqq n$, disjoint sets in $\mathscr{B}$ bounded away from zero, $M_{t}\left(B_{k}\right)$ $1 \leqq k \leqq n$ are independent random variables and so, 


$$
\begin{aligned}
E^{\omega} \exp \left[-\alpha \int_{(0, \infty)}\right. & \left.u M_{t}(d u)\right]=\lim _{n \rightarrow \infty} E^{\omega} \exp \left[-\alpha \sum_{k=1}^{n 2^{n-1}} \frac{k}{2^{n}} M_{t}\left[\left(\frac{k}{2^{n}}, \frac{k+1}{2^{n}}\right]\right]\right] \\
& =\lim _{n \rightarrow \infty} \prod_{k=1}^{n 2^{n}-1} E^{\omega} \exp \left[-\alpha \frac{k}{2^{n}} M_{t}\left[\left(\frac{k}{2^{n}}, \frac{k+1}{2^{n}}\right]\right]\right] \\
& =\lim _{n \rightarrow \infty} \exp \left[-\sum_{k=1}^{n 2^{n}-1}\left(1-e^{-\alpha\left(k / 2^{n}\right)}\right) \nu_{t}\left[\left(\frac{k}{2^{n}}, \frac{k+1}{2^{n}}\right]\right](\omega)\right] \\
& =\exp \left[-\int_{(0, \infty)}\left(1-e^{-\alpha u}\right) \nu_{t}(d u)(\omega)\right] .
\end{aligned}
$$

From this equation, we see that

$$
\int_{(0, \infty)}\left(1-e^{-\alpha u}\right) \nu_{t}(d u)(\omega)<\infty
$$

and this implies that $\nu_{t}(\cdot)(\omega)$ is a Levy measure.

\section{REFERENCES}

1. R. M. Blumenthal and R. K. Getoor, Markov Porcesses and Potential Theory, Academic Press, New York and London (1968).

2. L. Breiman, Probability, Addison-Wesley Publishing Company, Inc., Reading, Menlo Park and London (1968).

3. R. K. Getoor, Some remarks on continuous additive functionals. Ann. Math. Stat., 38 (1967).

4. R. K. Getoor and M. J. Sharpe, Last exit times and additive functionals, (to appear).

5. K. Ito, Stochastic processes, Aarhus. Lecture Notes Series No. 16 (1969).

6. B. Maisonneuve, Topologies du type de Skorohod, Seminaire de Probabilities VI Lecture Notes in Math. 258. Springer, Berlin, Heidelberg and New York (1972).

7. K. R. Parthasarathy, Probability on Metric Spaces, Academic Press, New York and London (1967).

Received March 22, 1974. The research for this article was supported by the National Research Council of Canada and la Direction Générale de l'Enseignement Supérieur du Québec.

MCGILL UNIVERSITY 


\section{PACIFIC JOURNAL OF MATHEMATICS}

\section{EDITORS}

RICHARD ARENS (Managing Editor)

University of California

Los Angeles, California 90024

\section{J. DugundJI}

Department of Mathematics University of Southern California Los Angeles, California 90007

D. Gilbarg and J. Milgram

Stanford University

Stanford, California 94305

\section{ASSOCIATE EDITORS}
E. F. BECKENBACH
B. H. NeumanN
F. WoLF
K. YoshiDA

\section{SUPPORTING INSTITUTIONS}

\author{
UNIVERSITY OF BRITISH COLUMBIA \\ CALIFORNIA INSTITUTE OF TECHNOLOGY \\ UNIVERSITY OF CALIFORNIA \\ MONTANA STATE UNIVERSITY \\ UNIVERSITY OF NEVADA \\ NEW MEXICO STATE UNIVERSITY \\ OREGON STATE UNIVERSITY \\ UNIVERSITY OF OREGON \\ OSAKA UNIVERSITY
}

\author{
UNIVERSITY OF SOUTHERN CALIFORNIA \\ STANFORD UNIVERSITY \\ UNIVERSITY OF TOKYO \\ UNIVERSITY OF UTAH \\ WASHINGTON STATE UNIVERSITY \\ UNIVERSITY OF WASHINGTON \\ AMERICAN MATHEMATICAL SOCIETY
}

The Supporting Institutions listed above contribute to the cost of publication of this Journal, but they are not owners or publishers and have no responsibility for its contents or policies.

Mathematical papers intended for publication in the Pacific Journal of Mathematics should be in typed form or offset-reproduced (not dittoed), double spaced with large margins. Underline Greek letters in red, German in green, and script in blue. The first paragraph or two must be capable of being used separately as a synopsis of the entire paper. Items of the bibliography should not be cited there unless absolutely necessary, in which case they must be identified by author and Journal, rather than by item number. Manuscripts, in duplicate, may be sent to any one of the four editors. Please classify according to the scheme of Math. Reviews, Index to Vol. 39. All other communications should be addressed to the managing editor, or Elaine Barth, University of California, Los Angeles, California, 90024.

100 reprints are provided free for each article, only if page charges have been substantially paid. Additional copies may be obtained at cost in multiples of 50 .

The Pacific Journal of Mathematics is issued monthly as of January 1966. Regular subscription rate: $\$ 72.00$ a year (6 Vols., 12 issues). Special rate: $\$ 36.00$ a year to individual members of supporting institutions.

Subscriptions, orders for back numbers, and changes of address should be sent to Pacific Journal of Mathematics, 103 Highland Boulevard, Berkeley, California, 94708.

PUBLISHED BY PACIFIC JOURNAL OF MATHEMATICS, A NON-PROFIT CORPORATION Printed at Jerusalem Academic Press, POB 2390, Jerusalem, Israel.

$$
\begin{gathered}
\text { Copyright (C) } 1975 \text { Pacific Journal of Mathematics } \\
\text { All Rights Reserved }
\end{gathered}
$$




\section{Pacific Journal of Mathematics}

\section{Vol. 58, No. $2 \quad$ April, 1975}

Zvi Artstein and John Allen Burns, Integration of compact set-valued functions . . . . . . . . . 297

Mark Benard, Characters and Schur indices of the unitary reflection group $[321]^{3} \ldots \ldots \ldots . .309$

Simeon M. Berman, A new characterization of characteristic functions of absolutely continuous

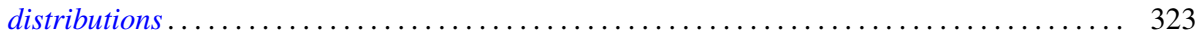

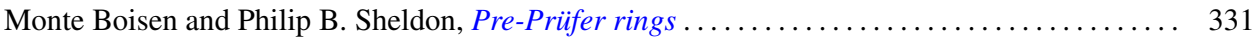

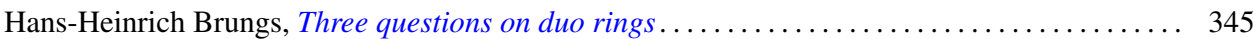

Iracema M. Bund, Birnbaum-Orlicz spaces of functions on groups................. 351

John D. Elwin and Donald R. Short, Branched immersions between 2-manifolds of higher

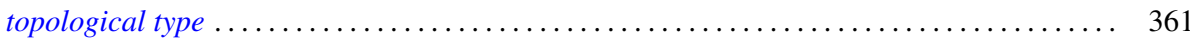

Eric Friedlander, Extension functions for rank 2, torsion free abelian groups . .......... 371

Jon Froemke and Robert Willis Quackenbush, The spectrum of an equational class of

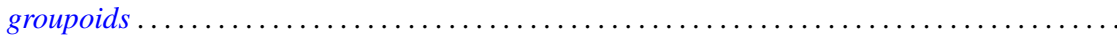

Barry J. Gardner, Radicals of supplementary semilattice sums of associative rings ...........

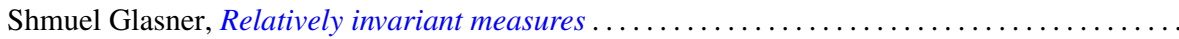

George Rudolph Gordh, Jr. and Sibe Mardesic, Characterizing local connectedness in inverse

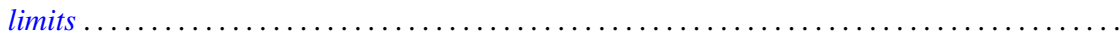

Siegfried Graf, On the existence of strong liftings in second countable topological spaces......

Stanley P. Gudder and D. Strawther, Orthogonally additive and orthogonally increasing

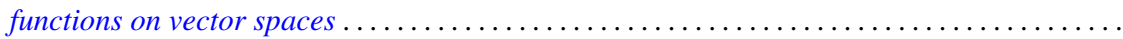

Darald Joe Hartfiel and Carlton James Maxson, A characterization of the maximal monoids and

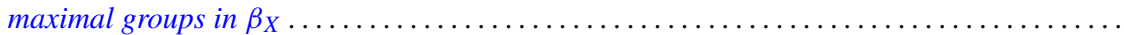

Robert E. Hartwig and S. Brent Morris, The universal flip matrix and the generalized faro-shuffle. .

William Emery Haver, Mappings between ANRs that are fine homotopy equivalences. .

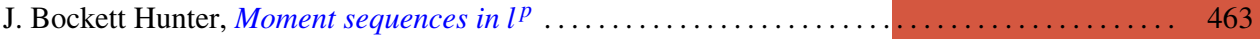

Barbara Jeffcott and William Thomas Spears, Semimodularity in the completion of a poset.... 467

Jerry Alan Johnson, A note on Banach spaces of Lipschitz functions . . . . . . . . . . . . 475

David W. Jonah and Bertram Manuel Schreiber, Transitive affine transformations on

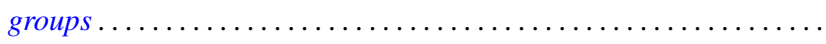

Karsten Juul, Some three-point subset properties connected with Menger's characterization of

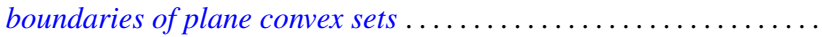

Ronald Brian Kirk, The Haar integral via non-standard analysis . . . . . . . . . . . . . 517

Justin Thomas Lloyd and William Smiley, On the group of permutations with countable

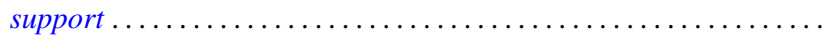

Erwin Lutwak, Dual mixed volumes .................................. 531

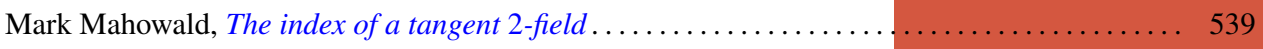

Keith Miller, Logarithmic convexity results for holomorphic semigroups . . . . . . . . . . . . 549

Paul Milnes, Extension of continuous functions on topological semigroups . . . . . . . . . . 553

Kenneth Clayton Pietz, Cauchy transforms and characteristic functions ................ 563

James Ted Rogers Jr., Whitney continua in the hyperspace $C(X) \ldots \ldots \ldots \ldots \ldots \ldots \ldots \ldots .569$

Jean-Marie G. Rolin, The inverse of a continuous additive functional . . . . . . . . . . . . 585

William Henry Ruckle, Absolutely divergent series and isomorphism of subspaces . ........ 605

Rolf Schneider, A measure of convexity for compact sets . ..................... 617

Alan Henry Schoenfeld, Continous measure-preserving maps onto Peano spaces .......... 627

V. Merriline Smith, Strongly superficial elements .......................... 643

Roger P. Ware, A note on quadratic forms over Pythagorean fields . . . . . . . . . . . . . . 651

Roger Allen Wiegand and Sylvia Wiegand, Finitely generated modules over Bezout rings . . . . 655

Martin Ziegler, A counterexample in the theory of definable automorphisms . . . . . . . . . 665 